\section{Totraumbestimmung nach Fowler bei Patienten mit Lungenemphysem mit Hilfe von $\mathrm{C}^{18} \mathrm{O}_{2}$}

T. Meyer ${ }^{1}$, H. Schulz ${ }^{1}$, P. Brand ${ }^{1}$, M. Kohlhäufl², J. Heyder ${ }^{1}$, K. Häußinger ${ }^{2}$

Klinische Kooperationsgruppe „Aerosolmedizin“ des ${ }^{1}$ GSF, Forschungszentrum für Umwelt und Gesundheit und den

${ }^{2}$ Asklepios Fachkliniken München-Gauting
Zusammenfassung: Patienten mit Lungenemphysem zeigen in der Lungenfunktionsdiagnostik neben Veränderungen der Lungenvolumina eine Zunahme des Atemwegswiderstandes $\left(R_{\text {tot }}\right)$, und eine Abnahme des Tiffeneau-Wertes $\left(\mathrm{FEV}_{1}\right)$. Als Ursache hierfür wird neben dem Bronchialkollaps auch die Obstruktion der Atemwege angenommen, die folglich zu einer Reduzierung des Totraumvolumens führen sollte. Es wurde eine Methode entwickelt und getestet, die es ermöglicht, die Totraummessung nach Fowler [2] bei Patienten mit Lungenemphysem durchzuführen. Um die Methode nach Fowler anwenden zu können, ist eine eindeutige Unterteilung eines Inertgas-Exspirogrammes in drei Bereiche notwendig. Während diese drei Bereiche im Exspirogramm eines Lungengesunden klar abgrenzbar sind (Abb. 1; Exspirogramm 3), ist diese Differenzierung bei Patienten mit Lungenemphysem wegen des fließenden Überganges der drei Bereiche nicht möglich (Abb. 1; Exspirogramm 2). Erst der Einsatz des Testgases $\mathrm{C}^{18} \mathrm{O}_{2}$ ermöglicht es auch bei Patienten mit Lungenemphysem, diese drei Bereiche klar voneinander zu trennen (Abb.1; Exspirogramm 1), womit die Grundlage für die zuverlässige Bestimmung des funktionellen Totraumes gegeben ist. In dieser Studie wurden 21 lungengesunde Nichtraucher und 29 Patienten mit Lungenemphysem untersucht. Es fand sich eine sehr gute Korrelation zwischen den Totraumvolumina, die aus den Ar-Exspirogrammen ermittelt wurden mit den Totraumvolumina aus den $\mathrm{C}^{18} \mathrm{O}_{2}$-Exspirogrammen (Abb. 2). Dies zeigt, dass die Bestimmung des funktionellen Totraumvolumens aus den $\mathrm{C}^{18} \mathrm{O}_{2}$-Exspirogrammen eine valide Methode ist. Es zeigte sich eine Korrelation zwischen Atemwegswiderstand und Totraumvolumen bei den Emphysematikern, nicht jedoch bei den Lungengesunden. Ein signifikanter Unterschied konnte auch zwischen diesen beiden Kollektiven bezüglich des Atemwegswiderstandes nachgewiesen werden $(0,20 \pm 0,10 \mathrm{kPa} / \mathrm{l} / \mathrm{s}$ vs. $0,49 \pm 0,27 \mathrm{kPa} / \mathrm{l} / \mathrm{s})$, es fand sich jedoch kein Unterschied bezüglich des Totraumvolumens (Tab.1). Es muss davon ausgegangen werden, dass bei Emphysematikern der Funktionsverlust von Alveolen im Bereich der respiratorischen Bronchioli zu einer Verschiebung der Grenze des funktionellen Totraumes führt. Die Erweiterung des funktionellen Totraumes in Bereiche der Lungenperipherie müsste folglich zu einer Vergrößerung des Totraumvolumens führen, wird jedoch durch die zentrale Obstruktion beim Lungenemphysem wieder kompensiert.
Measurement of the Fowler Dead Space in Patients with Lung Emphysema: In patients with lung emphysema, changes in lung volumes as well as changes in airway resistance are well known. The change in airway resistance is caused by obstruction of central airways, which is supposed to reduce the respiratory dead space. Until now, it was not possible to measure the respiratory dead space in patients with lung emphysema using the method of Fowler [2], because in this method distinction of the three phases of an inert gas expirogram is essential. While this distinction is easy in healthy subjects (fig. 1; expirogram 3), the separation of the three phases in patients with lung emphysema is not possible due to gradual transition of phase II into phase III in these patients (fig. 1; expirogram 2). The use of $\mathrm{C}^{18} \mathrm{O}_{2}$ as tracer gas allows to separate phase II and phase III even if the patients have severe emphysema (fig. 1; expirogram 1). $\mathrm{CO}_{2}$ labeled with the stable oxygen isotope ${ }^{18} \mathrm{O}\left(\mathrm{C}^{18} \mathrm{O}_{2}\right)$ is completely taken up in the gas exchanging region of the lung, but not from the conducting airways. Therefore $\mathrm{C}^{18} \mathrm{O}_{2}$ is only expired from the dead space of the lung, but not from the alveolar region. Hence, $\mathrm{C}^{18} \mathrm{O}_{2}$ allows exact measurement of the respiratory dead space in patients with lung emphysema. 21 healthy nonsmoking subjects and 29 patients with clinical signs of lung emphysema participated in this study. There was a good correlation between respiratory dead space, measured by the use of $\mathrm{Ar}$-gas and $\mathrm{C}^{18} \mathrm{O}_{2}$-gas in healthy subjects (fig. 2). This indicates, that the use of $\mathrm{C}^{18} \mathrm{O}_{2}$ is a valid method to measure the functional dead space. As expected, there was also a correlation between the airway resistance and respiratory dead space in patients with lung emphysema (fig. 3), but not in healty subjects There was no significant difference of the mean values of the respiratory dead space between these two groups ( $223 \pm 43 \mathrm{ml}$ in healthy subjects vs. $227 \pm 52 \mathrm{ml}$ in patients), even though there were large differences in airway resistance $(0.20 \pm 0.10 \mathrm{kPa} / \mathrm{I} / \mathrm{s}$ vs. $0.49 \pm 0.27 \mathrm{kPa} / \mathrm{l} / \mathrm{s})$. This may be due to a loss of alveolar function in the area of the terminal bronchioli, which is typical for emphysematous patients. This entails a shift of functional dead space towards lung periphery and therefore causes an increase of the volume of functional dead space. But this enlargement may be compensated by the volume reduction, caused by the airway obstruction. Hence, these two oppositional mechanisms may result in only minimal change of dead space volume. 


\section{Einleitung}

Bei der Beurteilung von pathophysiologischen Veränderungen im Bereich des intrapulmonalen Gastransportes ist die Kenntnis des Totraumvolumens von großer Bedeutung. Zur funktionellen Bestimmung des Totraumvolumens hat sich die Methode nach Fowler [2] etabliert, die allerdings voraussetzt, dass im Exspirogramm der Mischluftanteil (Phase II) vom alveolaren Plateau (Phase III) eindeutig differenziert werden kann und dass die Phase III eines Inertgas-Exspirogramms linear verläuft. Exspirogramme von Patienten mit Lungenemphysem sind jedoch u.a. dadurch charakterisiert, dass die Phasen II und III fließend ineinander übergehen und dass der Konzentrationsverlauf während der Phase III gekrümmt ist (vgl. Abb.1, Kurve 2). Somit ist also eine Bestimmung des Totraumes nach der Fowlerschen Methode bei Patienten mit Lungenemphysem bislang nicht möglich.

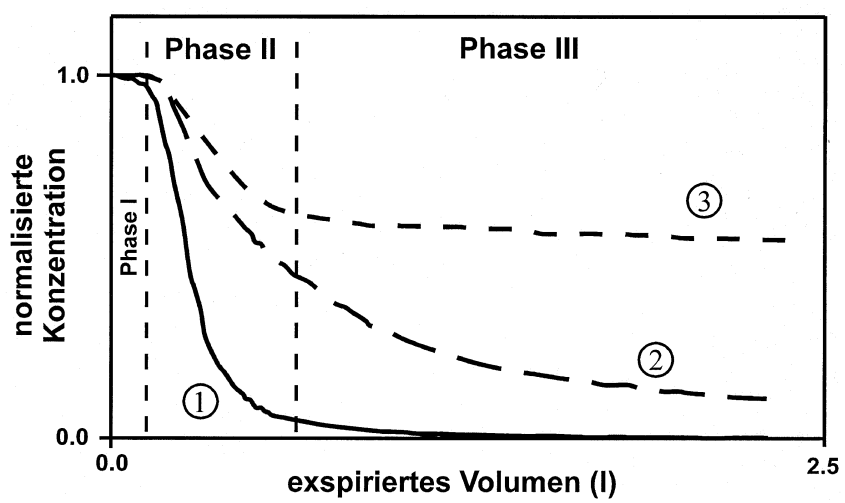

Abb.1 Beispiele von typischen Inertgas-Exspirogrammen. Kurve 1: $\mathrm{C}^{18} \mathrm{O}_{2}$-Exspirogramm eines Patienten mit Lungenemphysem. Kurve 2: Argon-Exspirogramm eines Patienten mit Lungenemphysem Kurve 3: Argon-Exspirogramm eines lungengesunden Nichtrauchers. Die drei Phasen eines Exspirogrammes lassen sich bei Kurve 1 und 3 deutlich voneinander differenzieren, wogegen dies bei Kurve 2 nicht möglich ist.

Um diese Problematik zu lösen, bietet sich der Einsatz von markiertem Kohlendioxid $\left(\mathrm{C}^{18} \mathrm{O}_{2}\right)$ als Testgas an. Wie in neueren Studien gezeigt werden konnte [2,5], wird das Testgas $\mathrm{C}^{18} \mathrm{O}_{2}$ im gasaustauschenden Bereich der Lunge vollständig absorbiert, jedoch nicht in den luftleitenden Atemwegen. Die Absorption des $\mathrm{C}^{18} \mathrm{O}_{2}$ erfolgt fast ausschließlich in Gegenwart des Enzyms Carboanhydrase der Erythrozyten. Somit kann $\mathrm{C}^{18} \mathrm{O}_{2}$ nur im stark durchbluteten Bereich der Lunge, dem Alveolarbereich absorbiert werden. Zwar konnte in tierexperimentellen Studien [6] gezeigt werden, dass $\mathrm{C}^{18} \mathrm{O}_{2}$ $\mathrm{zu}$ einem sehr geringen Teil auch von den luftleitenden Atemwegen absorbiert wird, dieser Anteil ist jedoch vernachlässigbar gering. Durch diese Eigenschaften fällt die massenspektrometrisch gemessene Konzentration bei der Exhalation von alveolarer Luft auf Null ab (vgl. Abb.1, Kurve 1). So kann durch den zusätzlichen Einsatz des Testgases $\mathrm{C}^{18} \mathrm{O}_{2}$ bei Patienten mit Lungenemphysem aus dem $\mathrm{C}^{18} \mathrm{O}_{2}$-Exspirogramm der Totraum nach der Fowlerschen Methode abgeleitet werden. Zusätzlich ermöglicht der Einsatz von $\mathrm{C}^{18} \mathrm{O}_{2}$ die exakte Trennung der Phase II von Phase III (vgl. Abb.1) und erlaubt damit die klare Zuordnung von exspirierter Luft aus den alveolaren Bereichen der Lunge. Dies ist von Bedeutung,

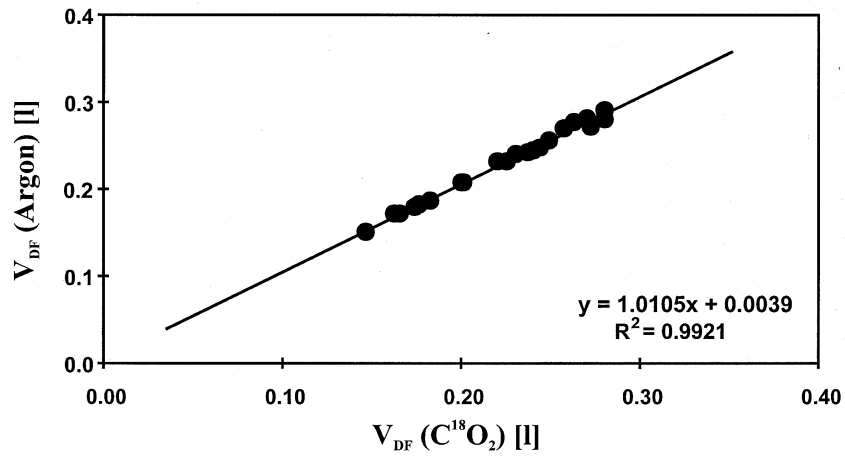

Abb. 2 Korrelation der Totraumvolumina, abgeleitet aus Argon- und $\mathrm{C}^{18} \mathrm{O}_{2}$-Exspirogrammen von lungengesunden Nichtrauchern.

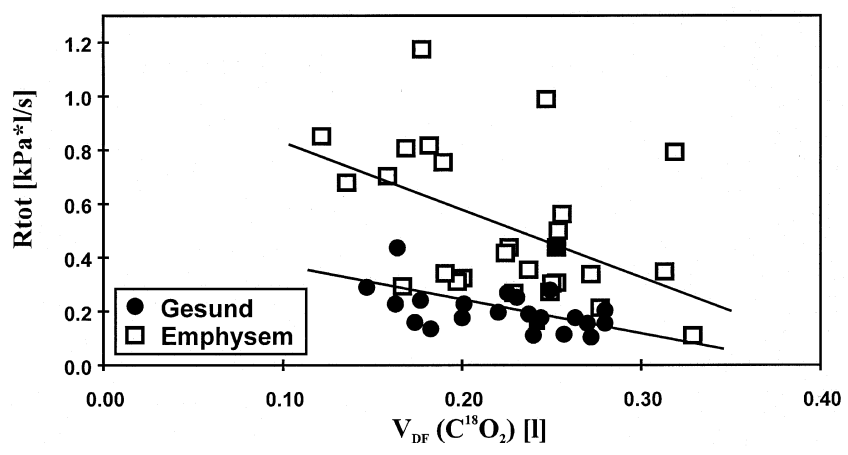

Abb. 3 Korrelationen zwischen Atemwegswiderstand $\mathrm{R}_{\text {tot }}$ und Totraumvolumen $\left(\mathrm{C}^{18} \mathrm{O}_{2}\right)$ bei lungengesunden Probanden und Patienten mit Lungenemphysem. Bei annähernd gleichem Totraumvolumen weisen die Kollektive unterschiedliche Atemwegswiderstände auf.

da eine Analyse der Phase III von Inertgas-Exspirogrammen wie z.B. Argon die Beurteilung der alveolaren Gasmischung erlaubt [4].

In dieser Studie sollte gezeigt werden, ob der Einsatz von $\mathrm{C}^{18} \mathrm{O}_{2}$ als Testgas beim Einatemzugmanöver geeignet ist, eine valide Totraumbestimmung nach Fowler bei Lungengesunden und bei Patienten mit Lungenemphysem durchzuführen.

\section{Methodik}

Patienten: Es wurden 21 lungengesunde Nichtraucher und 29 Patienten mit den klinischen und radiologischen Zeichen eines Lungenemphysems untersucht. Eine Übersicht über die Lungenfunktionswerte und das Alter gibt Tab. 1 wieder.

Einatemzugmanöver: Bei den Atemmanövern wurde ein Testgasgemisch verwendet, das bei normaler Sauerstoffkonzentration auch die Testgase Argon (Ar, $3 \%$ ) und $\mathrm{C}^{18} \mathrm{O}_{2}(0,2 \%)$ enthielt. Die Probanden und Patienten wurden in sitzender Position untersucht. Unter visueller Kontrolle der konstanten Atemstromstärke (Soll 0,5 l/s) wurde folgendes Atemmanöver vom Niveau der funktionellen Residualkapazität (FRC) aus begonnen: Die Inhalation erfolgte bis 90 \% TLC und unmittelbar anschließend, ohne Atempause, wurde bis unter das FRCNiveau exhaliert. Die Gaskonzentrationen wurden kontinuierlich direkt am Mundstück mit Hilfe eines Respirationsmassenspektrometers gemessen. 
Tab. 1

\begin{tabular}{lcccc}
\hline & Gesunde & \multicolumn{3}{c}{ Emphysematiker } \\
& & $\mathrm{V}_{\mathrm{DF}}$ & & $\mathrm{V}_{\mathrm{DF}}$ \\
\hline Alter [Jahre] & $37 \pm 11$ & & $66 \pm 10$ & \\
$\mathrm{R}_{\text {tot }}[\mathrm{kPa} / \mathrm{l} / \mathrm{s}]$ & $0,20 \pm 0,10$ & + & $0,49 \pm 0,27$ & + \\
$\mathrm{FEV}_{1}[\%$ Soll] & $106 \pm 15$ & + & $47 \pm 34$ & + \\
$\mathrm{MEF}_{75}[\%$ Soll] & $100 \pm 24$ & + & $29 \pm 28$ & + \\
$\mathrm{MEF}_{50}[\%$ Soll] & $84 \pm 25$ & & $27 \pm 40$ & \\
$\mathrm{MEF}_{25}[\%$ Soll] & $75 \pm 34$ & & $23 \pm 30$ & \\
$\mathrm{~V}_{\mathrm{DF}}[\mathrm{ml}]$ & $223 \pm 42$ & & $227 \pm 52$ & \\
$\mathrm{~V}_{\mathrm{DF}}(\mathrm{Ar})[\mathrm{ml}]$ & $230 \pm 43$ & + & - & - \\
\hline
\end{tabular}

$V_{D F}$ : Totraumvolumen ermittelt aus den $\mathrm{C}^{18} \mathrm{O}_{2}$-Exspirogrammen.

$\mathrm{V}_{\mathrm{DF}}(\mathrm{Ar})$ : Totraumvolumen ermittelt aus den Ar-Exspirogrammen.

+ gibt eine signifikante Korrelation $(p<0,05)$ zwischen $V_{D F}$ und dem jeweils betrachteten Parameter an

Respirationsmassenspektrometrie: Eine Probe der in- und exspirierten Luft wurde direkt am Mundstück durch eine beheizte $\left(37^{\circ} \mathrm{C}\right), 1,22 \mathrm{~m}$ lange Kapillare mit einem Fluss von $14 \mathrm{ml} / \mathrm{min}$ angesaugt und in einem Magnetsektor-Massenspektrometer (modifiziertes DLT 1100 R Dennis Leigh Technology Ltd.; GB) kontinuierlich analysiert. Die Tot- und Verzögerungszeiten wurden für alle Gase täglich gemessen und waren über den Zeitraum der Untersuchung konstant. Für $\mathrm{C}^{18} \mathrm{O}_{2}$ betrug die Totzeit $279 \pm 2 \mathrm{~ms}$, die Verzögerungszeit ( $5 \%-95 \%$ ) lag bei $28 \pm 2 \mathrm{~ms}$. Entsprechende Werte wurden auch für Argon gemessen. Mit Hilfe eines analogen 10-KanalRechners nach Scheidt und Slama [5] wurden unerwünschte Effekte durch Wasserdampf und Absolutdruckschwankungen kompensiert, in dem die Summe aller gemessenen respiratorischen Gase auf 1 normiert wurde und so rechnerisch die fraktionellen Konzentrationen ermittelt wurden. Das Gas $\mathrm{C}^{18} \mathrm{O}_{2}$ wurde bei einem Masse-Ladungs-Verhältnis von 48 gemessen, der Abstand zu den benachbarten Gasen beträgt $500: 1$.

Eigenschaften der verwendeten Gase: Argon hat eine Atomgewicht von $40, \mathrm{C}^{18} \mathrm{O}_{2}$ von 48. Somit haben beide Gase annähernd gleiche diffusible Eigenschaften ( $\mathrm{Ar}$ diffundiert in Luft ca. 1,09-mal schneller als $\mathrm{C}^{18} \mathrm{O}_{2}$ ), werden jedoch unterschiedlich schnell vom Gewebe aufgenommen. $\mathrm{C}^{18} \mathrm{O}_{2}$ gehört wegen seiner guten Wasserlöslichkeit - wie auch das „normale“ Kohlendioxid, $\mathrm{C}^{16} \mathrm{O}_{2}-\mathrm{zu}$ den am schnellsten vom Gewebe aufgenommenen Gasen (ca. 20-mal schneller als z.B. $\mathrm{O}_{2}$ oder Ar). Aufgrund der unterschiedlichen physikalischen Eigenschaften eignet sich somit $\mathrm{C}^{18} \mathrm{O}_{2}$ besser zur Bestimmung des Totraumvolumens, weil die Grenze zwischen gut durchblutetem Alveolarbereich und schlechter durchblutetem Bronchialbereich schärfer abgrenzbar ist. Zur Beurteilung von intrapulmonalen Gasmischungsprozessen ist Argon wegen seiner schlechteren Löslichkeit vorzuziehen.

Datenaufzeichnung und Auswertung: Die kompensierten Gassignale und das Signal für die Atemstromstärke wurden mit einer Abtastrate von $500 \mathrm{~Hz}$ digitalisiert und von einem PC gespeichert. Vor der rechnergestützten Analyse wurden die Exspirogramme zeitlich um die aktuelle Totzeit korrigiert und schließlich als Funktion des exspirierten Volumens dargestellt. Aus diesen normierten $\mathrm{C}^{18} \mathrm{O}_{2}$-Exspirogrammen wurde schließlich nach der Fowlerschen Methode [2] der Totraum bestimmt, den Fowler bereits 1948 als „physiologischen Totraum" bezeichnet hat. Bei Lungengesunden wurde nach derselben Methode zusätzlich auch der Totraum aus den Argon-Exspirogrammen ermittelt.

Reproduzierbarkeit der Daten: Von allen Messungen wurden Doppelbestimmungen gemacht. Die mittlere Abweichung der Einzelmesswerte lag bei der Bestimmung des Totraumvolumens mittels $\mathrm{C}^{18} \mathrm{O}_{2}$ bei Gesunden bei 2,7\% und bei Emphysematikern bei $5,3 \%$.

\section{Ergebnisse}

Die Ergebnisse aus der konventionellen Lungenfunktionsuntersuchung und aus dem Einatemzugmanöver sind z.T. in Tab. 1 aufgetragen. Wie zu erwarten, zeigten die Patienten mit Lungenemphysem bodyplethysmographisch ein erhöhtes intrathorakales Gasvolumen (ITGV) mit $207 \pm 38 \%$ Soll gegenüber $129 \pm 35 \%$ Soll bei Gesunden. Auch war der Quotient RV/ TLC bei den Emphysematikern mit 55,4 \pm 13,5 \% gegenüber $24,6 \pm 5,1 \%$ bei den Gesunden deutlich erhöht. Als Ausdruck der zentralen und peripheren Obstruktion wiesen Atemwegswiderstand $\left(\mathrm{R}_{\text {tot }}\right)$ sowie maximaler exspiratorischer Fluss (PEF) und Tiffeneau-Test $\left(\mathrm{FEV}_{1}\right)$ deutlich pathologische Werte auf (vgl. Tab.1). Der verminderte alveolar-kapilläre Gasaustausch spiegelte sich in einer auf $80 \%( \pm 31 \%)$ abgefallenen Diffusionskapazität wider.

Das aus dem $\mathrm{C}^{18} \mathrm{O}_{2}$-Exspirogramm abgeleitete Totraumvolumen betrug bei Gesunden $223 \pm 42 \mathrm{ml}$. Die Korrelation zwischen den aus den Argon- bzw. $\mathrm{C}^{18} \mathrm{O}_{2}$-Exspirogrammen abgeleiteten Totraumvolumina ist für Gesunde in Abb. 2 grafisch dargestellt. Man erkennt, dass es sich hierbei um eine eindeutige Beziehung handelt, was sich in einem $\mathrm{R}^{2}$ von 0,99 widerspiegelt $\left(\mathrm{V}_{\mathrm{DF}}[\mathrm{Ar}]=1,01 \cdot \mathrm{V}_{\mathrm{DF}}\left[\mathrm{C}^{18} \mathrm{O}_{2}\right]+0,004 \mathrm{l}\right)$. Jedoch war das Totraumvolumen aus den Argon-Exspirogrammen $\left(\mathrm{V}_{\mathrm{DF}}\right)$ im Mittel um $7 \mathrm{ml}$ größer $(\mathrm{p}<0,001)$ als das aus den $\mathrm{C}^{18} \mathrm{O}_{2}$-Exspirogrammen ermittelte.

Das aus den $\mathrm{C}^{18} \mathrm{O}_{2}$-Exspirogrammen ermittelte Totraumvolumen war bei Lungengesunden unerwarteterweise gleich groß wie bei Emphysematikern. Dieses Ergebnis steht scheinbar im Widerspruch zu den oben erwähnten, bodyplethysmographischen Parametern, die bei Emphysematikern im Sinne einer Bronchokonstriktion verändert waren und daher kleinere Totraumvolumina erwarten ließen. Für beide untersuchten Kollektive wurden daher die Korrelationen zwischen dem aus dem $\mathrm{C}^{18} \mathrm{O}_{2}$-Exspirogramm abgeleiteten Totraumvolumen und dem Atemwegswiderstand betrachtet (vgl. Abb.3). Bei den Emphysematikern ließ sich eine signifikante Korrelation zwischen beiden Parametern nachweisen $(p=0,015$ nach Pearson), nicht jedoch bei den Lungengesunden ( $p=0,74)$.

\section{Diskussion}

Aus den Exspirogrammen ist erkennbar, dass der Konzentrationsverlauf von $\mathrm{C}^{18} \mathrm{O}_{2}$ während der Phase I keine Steigung aufweist. Dies muss als Bestätigung interpretiert werden, dass in den luftleitenden Atemwegen dieses Testgas nicht oder nur zu einem vernachlässigbar geringem Anteil absorbiert wird. 
Die Ergebnisse zeigen, dass die funktionelle Totraumbestimmung aus dem $\mathrm{C}^{18} \mathrm{O}_{2}$-Exspirogramm nach der von Fowler beschriebenen Methode bei den gesunden Probanden identische Ergebnisse liefert wie aus dem Argon-Exspirogramm abgeleitete Werte.

Die eingangs erwähnten besonderen Eigenschaften von $\mathrm{C}^{18} \mathrm{O}_{2}$ und die bei den Gesunden gefundene enge Korrelation zwischen Argon- und $\mathrm{C}^{18} \mathrm{O}_{2}$-Totraumvolumen legt nahe, dass der hier vorgestellte experimentelle Ansatz auch beim Patienten mit Lungenemphysem valide Ergebnisse liefert. Die Analyse von $\mathrm{C}^{18} \mathrm{O}_{2}$-Exspirogrammen stellt somit bei Patienten mit Lungenemphysem derzeit die einzige verfügbare Methode zur Totraumbestimmung dar.

Da der klinische Übergang zwischen Lungengesunden und Emphysematikern fließend ist, würde man auch einen fließenden Übergang der gemessenen Totraumvolumina und den flussdynamischen Parametern zwischen diesen beiden Kollektiven erwarten.

Während bei Patienten mit Lungenemphysem zwischen den beiden Parametern Totraumvolumen und Atemwegswiderstand eine signifikante Korrelation besteht $(p=0,015)$, ist kein Zusammenhang zwischen diesen beiden Parametern beim Lungengesunden nachweisbar (vgl. Abb.3). Diese Beobachtung kann mit dem Gesetz von Hagen-Poiseuille erklärt werden, wonach der Strömungswiderstand in einer Röhre in der 4. Potenz vom Durchmesser abhängig ist. Somit beeinflussen geringfügige Änderungen der physiologisch weiten Atemwegsdurchmesser beim Gesunden den Atemwegswiderstand nur wenig, während beim Patienten mit bereits obstruktiven Atemwegen jede weitere, geringfügige Änderung den Atemwegswiderstand stärker beeinflusst.

Aus Abb. 3 und Tab. 1 geht jedoch hervor, dass die beiden Kollektive nicht wie oben postuliert entlang einer Funktion der 4. Potenz fließend ineinander übergehen, sondern dass die beiden Kollektive bei gleichen Totraumvolumina verschiedene Atemwegswiderstände aufweisen. Es stellt sich somit die Frage, weshalb die Patienten erhöhte Atemwegswiderstände aufweisen, obwohl die Totraumvolumina gegenüber den Gesunden gleich sind. Bei dem von uns untersuchten Patientenkollektiv handelte es sich fast ausschließlich um Raucher. Es ist die vorherrschende Lehrmeinung, dass Raucher überwiegend die zentrialobuläre Form des Emphysems entwickeln [1]. Somit ist vorwiegend mit einem Funktionsverlust der Alveolen im Bereich der respiratorischen Bronchioli zu rechnen. Da die destruierten Alveoli nicht mehr am Gasaustausch beteiligt sind, wandert auch die funktionelle Grenze zwischen konduktiven Atemwegen und gasaustauschendem Bereich nach peripher, das funktionelle, aus dem $\mathrm{C}^{18} \mathrm{O}_{2}$-Exspirogramm abgeleitete Totraumvolumen wird also in den gasaustauschenden Bereich der Lunge verschoben. Bei der Bestimmung des Totraumes mit unserer Methode wird wie eingangs beschrieben $-\mathrm{C}^{18} \mathrm{O}_{2}$ nur in den gasaustauschenden Bereichen der Lunge vollständig absorbiert. Im Exspirogramm führt dies ebenfalls zu einer Verschiebung der Grenze zwischen Phase II und Phase III. Der gemessene Totraum wird also größer. Auf der Grundlage des Lungenmodells von Weibel [7] würde eine Verschiebung dieser Grenze zwischen Phase II und Phase III um nur eine Atemwegsgeneration nach peripher (von Generation 17 nach 18) rechnerisch zu einer Zunahme des Totraumvolumens von $61,1 \mathrm{ml}$ führen. Nach Weibel sind im Bereich der 17. Atemwegsgeneration $12 \%$ und im Bereich der 18 . Atemwegsgeneration bereits $25 \%$ der Atemwegsoberfläche alveolarisiert. Eine Destruktion der Alveolen in diesen Regionen führt also zu einer Verschiebung der Grenze des funktionellen Totraumes. Ebenso führt die verminderte Alveolenzahl zu einer verlangsamten Absorption des $\mathrm{C}^{18} \mathrm{O}_{2}$ und so zu einer messbaren Verschiebung des Übergangs Phase II zu III im Exspirogramm.

Es können also bei Patienten mit Lungenemphysem zwei voneinander unabhängige Effekte das funktionell bestimmte Totraumvolumen verändern: 1. zentrale Bronchokonstriktionen führen zu einer Reduktion des Totraumvolumens und 2. die Verschiebung der stationären Front in die Lungenperipherie führt zu einer Zunahme des Totraumvolumens. Bei Patienten mit Lungenemphysem führt offensichtlich die Überlagerung dieser beiden Mechanismen dazu, dass das Totraumvolumen in der Summe unverändert bleibt.

\section{Literatur:}

${ }^{1}$ American Thoracic Society . Standards for the diagnosis and care of patients with chronic obstructive pulmonary. Am Rev Respir Dis 1987; 44: 136 - 225

${ }^{2}$ Fowler WS. Lung function studies. II. The respiratory dead space. Am J Physiol 1948; 154: 405 - 416

${ }^{3}$ Meyer T, Schulz H, Brand P, Selzer T, App E, Fruhmann G, Heyder J. $\mathrm{C}^{18} \mathrm{O}_{2}$ erlaubt die Differenzierung von Mischluftanteil und alveolarem Plateau im Exspirogramm von Patienten mit Lungenemphysem. Atemw Lungenkrkh 1996; 22: 328 - 330

${ }^{4}$ Paiva M, Engel LA (Hrsg). Gas mixing in the lung periphery. In: Chang HK und Paiva M. Respiratory Physiology. An analytical approach. New York: Marcel Dekker, 1989: 245-276

${ }^{5}$ Scheid P, Slama H, Piiper J. Elektronische Kompensation der Effekte des Wasserdampfes in der Massenspektrometrie. Pneumologie 1971; 144: 279 - 280

${ }^{6}$ Schulz H, Meyer T, Ziesenis A, Schulz A, Heyder J. Einsatz eines neuen Testgases $\left(\mathrm{C}^{18} \mathrm{O}_{2}\right)$ beim Einatemzug-Manöver zur Charakterisierung des Mischluftanteils (Phase II) im Exspirogramm. Atemw Lungenkrkh 1996; 22: 330 - 331

${ }^{7}$ Weibel ER (Hrsg). Morphometry of the human lung. Berlin: Springer, 1963

\section{Dr. Thomas Meyer}

Klinische Kooperationsgruppe „Aerosolmedizin“ Robert-Koch-Allee 6 82131 Gauting 